\title{
Special boundedness properties in numerical initial value problems
}

\author{
W. Hundsdorfer*, A. Mozartova ${ }^{\dagger}$ and M.N. Spijker ${ }^{\ddagger}$
}

August 12, 2010

\begin{abstract}
For Runge-Kutta methods, linear multistep methods and other classes of general linear methods much attention has been paid in the literature to important nonlinear stability properties known as total-variation-diminishing (TVD), strong stability preserving (SSP) and monotonicity. Stepsize conditions guaranteeing these properties were studied by Shu \& Osher (1988) and in numerous subsequent papers. Unfortunately, for many useful methods it has turned out that these properties do not hold. For this reason attention has been paid in the recent literature to the related and more general properties called total-variation-bounded (TVB) and boundedness.

In the present paper we focus on stepsize conditions guaranteeing boundedness properties of a special type. These boundedness properties are optimal, and distinguish themselves also from earlier boundedness results by being relevant to sublinear functionals, discrete maximum principles and preservation of nonnegativity. Moreover, the corresponding stepsize conditions are more easily verified in practical situations than the conditions for general boundedness given thus far in the literature.

The theoretical results are illustrated by application to the two-step Adams-Bashforth method and a class of two-stage multistep methods.

Key words. initial value problem, method of lines (MOL), ordinary differential equation (ODE), general linear method (GLM), total-variation-diminishing (TVD), strong-stability-preserving (SSP), monotonicity, total-variation-bounded (TVB), boundedness.
\end{abstract}

AMS subject classifications. 65L05, 65L06, 65L20, 65M20.

\section{Introduction}

\subsection{Bounds for numerical approximations}

In this paper we deal with the numerical solution of initial value problems of the form

$$
\frac{d}{d t} u(t)=F(t, u(t)) \quad(t \geq 0), \quad u(0)=u_{0}
$$

We shall study a wide class of numerical methods, for solving (1.1), via the analysis of an abstract generic numerical process of the type

$$
y_{i}=\sum_{j=1}^{l} s_{i j} x_{j}+\Delta t \cdot \sum_{j=1}^{m} t_{i j} F_{j}\left(y_{j}\right) \quad(1 \leq i \leq m) .
$$

Here $\Delta t>0$ denotes the stepsize, the vectors $x_{j}(1 \leq j \leq l)$ are the input vectors of the process, and $y_{i}(1 \leq i \leq m)$ the output vectors. In applications to concrete numerical

*CWI, P.O. Box 94079, 1090-GB Amsterdam, The Netherlands (willem.hundsdorfer@cwi.nl).

$\dagger$ CWI, P.O. Box 94079, 1090-GB Amsterdam, The Netherlands (a.mozartova@cwi.nl). Work of this author is supported by a grant from the Netherlands Organisation for Scientific Research NWO.

${ }_{\ddagger}^{\ddagger}$ Mathematical Institute, Leiden University, P.O. Box 9512, 2300-RA Leiden, The Netherlands (spijker@math.leidenuniv.nl). 
methods, the output vectors usually stand for approximations to the exact solution $u(t)$ of (1.1) at certain time levels $\bar{t}_{i}$, that is, $y_{i} \approx u\left(\bar{t}_{i}\right)(1 \leq i \leq m)$, and $F_{i}\left(y_{i}\right)=F\left(\bar{t}_{i}, y_{i}\right)$.

Process (1.2) is highly relevant to the important and very large class of general linear methods (GLMs), introduced by Butcher [1], cf. also e.g. Butcher [2, 3], Hairer \& Wanner [7], Hairer, Nørsett \& Wanner [8]. This class comprises, e.g., all Runge-Kutta methods, linear multistep methods and multistep-multistage variants thereof.

We can represent $N \geq 1$ consecutive steps of any GLM canonically by a process of type (1.2) with $m=N(s+r)$, where $s$ is the number of internal stages and $r$ the number of external stages computed at each step of the GLM. In this situation, the vectors $x_{j}(1 \leq i \leq$ l) stand for the starting vectors of the GLM, whereas the vectors $y_{i}(1 \leq i \leq m)$ represent the $N \cdot s$ internal and $N \cdot r$ external stage approximations computed during the $N$ steps. Furthermore, the parameter matrices $S=\left(s_{i j}\right) \in \mathbb{R}^{m \times l}, T=\left(t_{i j}\right) \in \mathbb{R}^{m \times m}$, corresponding to (1.2), are determined by the number of steps $N$ as well as by the coefficients of the given GLM. Detailed examples of such representations, as well as alternative representations of actual multistep-multistage methods, can be found in Spijker [22] for $N=1$ and in Hundsdorfer, Mozartova \& Spijker [13] for $N>1$; cf. also Section 4 of the present paper.

We denote by $\mathbb{V}$ the vector space on which the differential equation is defined, and by $\|\cdot\|$ a real functional on $\mathbb{V}$, i.e. $\|v\| \in \mathbb{R}$ for all $v \in \mathbb{V}$. In the rest of the present section, we assume $\|\cdot\|$ to be a convex functional, i.e.

$$
\|\lambda v+(1-\lambda) w\| \leq \lambda\|v\|+(1-\lambda)\|w\| \quad(\text { for } 0 \leq \lambda \leq 1 \text { and } v, w \in \mathbb{V}) .
$$

In applications, $\|\cdot\|$ will often be a norm or seminorm, see (2.9) below. But, more general convex functionals are useful as well, notably in connection with discrete maximum principles and preservation of nonnegativity; cf. e.g. Spijker [22] and Section 3.4 of the present paper.

For the general process (1.2), as well as for special instances thereof, much attention has been paid in the literature to the derivation of suitable upper bounds for $\left\|y_{i}\right\|$, in terms of the input vectors $x_{j}$, under the assumption that for given $\tau_{0}>0$

$$
\left\|v+\tau_{0} F_{i}(v)\right\| \leq\|v\| \quad(\text { for } 1 \leq i \leq m, \text { and } v \in \mathbb{V}) ;
$$

cf. e.g. Ferracina \& Spijker [4], Gottlieb, Ketcheson \& Shu [5], Gottlieb, Shu, \& Tadmor [6], Higueras [9, 10], Hundsdorfer \& Ruuth [14, 15], Hundsdorfer, Ruuth \& Spiteri [16], Shu \& Osher [20], Spijker [22].

In most papers, the focus has been on the situation where (1.2) stands for just one step $(N=1)$ of a GLM and

$$
s_{i 1}+s_{i 2}+\cdots+s_{i l}=1 \quad(1 \leq i \leq m) .
$$

Under assumption (1.5), the neat bound

$$
\left\|y_{i}\right\| \leq \max _{1 \leq j \leq l}\left\|x_{j}\right\| \quad(\text { for } 1 \leq i \leq m)
$$

has been studied extensively. Process (1.2) has been called monotonic or strongly stable (for given stepsize $\Delta t$, vector space $\mathbb{V}$, functional $\|\cdot\|$ and functions $F_{i}: \mathbb{V} \rightarrow \mathbb{V}$ ) if inequality (1.6) holds whenever $x_{i}$ and $y_{i}$ satisfy (1.2). Algebraic characterizations were derived of stepsize-coefficients $\gamma$ with the following important property:

Condition $0<\Delta t \leq \gamma \cdot \tau_{0}$ implies monotonicity, whenever $\mathbb{V}$ is a vector space, $\|\cdot\|$ a convex functional on $\mathbb{V}$, and the functions $F_{i}: \mathbb{V} \rightarrow \mathbb{V}$ satisfy (1.4);

see e.g. Spijker [22] and the references therein.

Unfortunately, for many useful GLMs there exists no $\gamma>0$ such that (1.7) holds, when one step of the method $(N=1)$ is represented in the form (1.2); some examples are given in Section 4 of this paper. Furthermore, in important cases where (1.2) stands for $N>1$ consecutive applications of a GLM, not even assumption (1.5) is fulfilled; cf. Section 4. 
These difficulties have led various authors to study bounds for $\left\|y_{i}\right\|$ that differ from (1.6) by a factor $\mu \geq 1$, i.e.

$$
\left\|y_{i}\right\| \leq \mu \cdot \max _{1 \leq j \leq l}\left\|x_{j}\right\| \quad(\text { for } 1 \leq i \leq m) .
$$

Such general bounds are formally weaker than (1.6) but still useful because they can reveal essential boundedness properties of the numerical methods under consideration, like the property of being total-variation bounded - for this important concept see e.g. LeVeque [18]. Stepsize conditions corresponding to general bounds (1.8) were derived, e.g., in Ruuth \& Hundsdorfer [19], Hundsdorfer, Mozartova \& Spijker [13].

The general bounds obtained thus far in the literature are relevant in cases where (1.7) is violated or even (1.5) is not in force. On the other hand, these bounds suffer still from the following two inconveniences: (1) the corresponding stepsize conditions, of type $0<\Delta t \leq \gamma \cdot \tau_{0}$, involve complicated conditions on $\gamma$ which are often difficult to check in practise; (2) the general bounds are relevant to seminorms but not to any wider class of functionals satisfying (1.3).

\subsection{Scope of the paper}

The main purpose of the present paper is to establish stepsize conditions guaranteeing special bounds for the generic process (1.2), thereby circumventing the two inconveniences just mentioned above. We shall find special bounds which can still be present in cases where (1.5) or (1.7) is violated and which are best possible in a definite sense. Moreover, these special bounds are relevant to a class of functionals $\|\cdot\|$ that is wider than the class of seminorms. Finally, and most importantly in view of applications, the corresponding stepsize conditions $0<\Delta t \leq \gamma \cdot \tau_{0}$ involve a condition on $\gamma$ which is easier to check in practise than the conditions relevant to the general bounds given in the literature.

In Section 2 of this paper, we review and extend bounds and monotonicity results for process (1.2), as given thus far in the existing literature. In Section 2.2, we first give a brief review of known monotonicity results for process (1.2). Next we consider a property which is a-priori more refined than pure monotonicity and we characterize in Theorem 2.4 stepsize conditions guaranteeing this property. In Section 2.3, we specify two generalizations of (1.6) which are relevant to process (1.2) in cases where (1.5) need not be fulfilled. Theorem 2.5 characterizes stepsize conditions guaranteeing these generalizations.

Section 3 contains the main theoretical findings of the paper. In Section 3.1, we formulate explicitly, for process (1.2), the special bounds mentioned above (for $\left\|y_{i}\right\|$ in terms of $\left.\left\|x_{j}\right\|\right)$, and mention three features which distinguish them from more general standard bounds (1.8). In Section 3.2, we study, in the situation of these special bounds, the characterizations provided by Theorem 2.5. We find the simplified version (3.4)-(3.7) of these characterizations. In Section 3.3, we study the special bounds for the case of seminorms $\|\cdot\|$; we find that these bounds are best possible in the sense specified by Theorem 3.4. The main theorem of Section 3.3, Theorem 3.5, gives simplified criteria for stepsize conditions guaranteeing the special bounds. Section 3.4 deals with the special bounds for the case of a natural class of functionals - the so-called sublinear functionals - which is essentially larger than the class of seminorms. Theorem 3.8 reveals the surprising fact that the special bounds are the only bounds which make sense in the context of general sublinear functionals. The main theorem of Section 3.4, Theorem 3.9, gives among other things a mild condition under which the simple condition (2.3) characterizes stepsize conditions guaranteeing the special bounds for sublinear functionals.

In Section 4 we illustrate the significance of the special boundedness theory by applying it to some concrete numerical methods. For most of these methods, the monotonicity results, as given in the literature, see e.g. [5, 22], are not (directly) applicable. Moreover, the boundedness theory, as given e.g. in [13] would lead to very complicated conditions. In Section 4.2 we study the two-step Adams-Bashforth method. When writing one step of the method in a standard fashion as a process of type (1.2), there is no $\gamma>0$ such that 
the monotonicity property (1.7) is present. But, by writing $N \geq 1$ steps of the method judiciously in the generic form (1.2), it turns out that Theorems 3.5, 3.9 yield conclusions which can nicely be interpreted in terms of boundedness and nonnegativity preservation of the method. In Section 4.3 we analyse a large class of $k$-step methods, containing both predictor-corrector methods and hybrid multistep methods. The monotonicity results, known from the literature, are not valid for many popular schemes of this class. By applying Theorem 3.9, we will show that for many methods of practical interest relevant boundedness properties are valid.

\section{Reviewing and extending results from the literature}

\subsection{Preliminaries}

Let $I$ stand for the identity matrix of order $m$, and let $S=\left(s_{i j}\right), T=\left(t_{i j}\right)$ denote the coefficient matrices corresponding to (1.2). For values $\gamma>0$ such that

$$
I+\gamma T \text { is invertible, }
$$

we write, similarly as in [13], [22],

$$
P=\left(p_{i j}\right)=(I+\gamma T)^{-1}(\gamma T), \quad R=\left(r_{i j}\right)=(I+\gamma T)^{-1} S .
$$

In the following we will always assume (2.1). To show that this assumption can be made with no loss of generality, we formulate the following lemma, which is an analogue of a result from [22, Lemma 4.2]. The proof of this lemma is compact, so we repeat it here.

Lemma 2.1 (Invertibility of $I+\gamma T$ ). Let $\tau_{0}>0, \gamma>0$ be given and $\Delta t=\gamma \cdot \tau_{0}$. Let $\mathbb{V}=\mathbb{R},\|\cdot\|=|\cdot|$ and assume $\mu$ is a constant such that (1.8) holds whenever $F_{i}: \mathbb{V} \rightarrow \mathbb{V}$ fulfil (1.4) and $y_{i}, x_{j} \in \mathbb{V}$ satisfy (1.2). Then (2.1) holds.

Proof. Let $\eta=\left[\eta_{i}\right] \in \mathbb{R}^{m}$ such that $(I+\gamma T) \eta=0$. We shall prove $\eta=0$.

We define $F_{i}(v)=-\left(1 / \tau_{0}\right) v$ (for all $v \in \mathbb{V}$ ), so that (1.4) is fulfilled with $\|\cdot\|=|\cdot|$. Clearly, (1.2) is satisfied, with $\Delta t=\gamma \cdot \tau_{0}$, by the vectors $x_{i}=0(1 \leq i \leq l)$ and $y_{i}=\eta_{i}(1 \leq i \leq m)$. By applying (1.8), there follows $\left|\eta_{i}\right|=\left|y_{i}\right| \leq \mu \cdot \max _{j}\left|x_{j}\right|=0$, therefore $\eta=0$.

We consider the following simple condition on $\gamma$ :

$$
P \geq 0, \quad R \geq 0
$$

These two inequalities - as well as any other inequalities between matrices appearing below - should be interpreted entry-wise. Condition (2.3) will play a prominent part in the following.

\subsection{Monotonicity with arbitrary convex functionals $\|\cdot\|$}

We recall shortly some concepts and results from the literature which are related to the monotonicity property (1.7). The next two theorems follow directly from [22, Theorems 2.2 , $2.4]$.

Theorem 2.2 (Criterion for monotonicity with arbitrary convex functional $\|\cdot\|$ ). Assume (1.5) and let $\gamma>0$. Then process (1.2) has the monotonicity property (1.7) if and only if $\gamma$ satisfies condition (2.3).

In the following, we use, for any given matrix $A=\left(a_{i j}\right)$, the notation $\operatorname{Inc}(A)$ to denote the incidence matrix of $A$, given by

$$
\operatorname{Inc}(A)=\left(\hat{a}_{i j}\right), \quad \text { where } \hat{a}_{i j}=1 \quad\left(\text { if } a_{i j} \neq 0\right), \hat{a}_{i j}=0 \quad\left(\text { if } a_{i j}=0\right) .
$$


Theorem 2.3 (Conditions on $S, T$ ). Assume (1.5). Then there is a $\gamma>0$ satisfying (2.3) if and only if $S \geq 0, T \geq 0, \operatorname{Inc}(T S) \leq \operatorname{Inc}(S)$ and $\operatorname{Inc}\left(T^{2}\right) \leq \operatorname{Inc}(T)$.

Clearly, for given matrices $S, T$, it is rather easy, by applying Theorems 2.2 and 2.3 to see whether there is a $\gamma>0$ such that (1.7) holds.

Under conditions (1.5), (2.3), we shall prove an interesting variant of (1.6), viz.

$$
\left\|y_{i}\right\| \leq \sum_{j=1}^{l}\left|s_{i j}\right|\left\|x_{j}\right\| \quad(1 \leq i \leq m) .
$$

Note that, when all $s_{i j}$ are nonnegative, the last bound is of particular interest because it is more refined and gives, in general, more information than (1.6). Clearly, all $s_{i j}$ are nonnegative as soon as (1.7) holds for some $\gamma>0$; cf. Theorems 2.2, 2.3.

We shall say that process (1.2) satisfies the bound (2.4) (for given stepsize $\Delta t$, vector space $\mathbb{V}$, functional $\|\cdot\|$ and functions $F_{i}: \mathbb{V} \rightarrow \mathbb{V}$ ), if (2.4) holds whenever $x_{i}$ and $y_{i} \in \mathbb{V}$ satisfy (1.2). The following property is an obvious variant of (1.7).

Condition $0<\Delta t \leq \gamma \cdot \tau_{0}$ implies the bound (2.4), whenever $\mathbb{V}$ is a vector space, $\|\cdot\|$ a convex functional on $\mathbb{V}$, and the functions $F_{i}: \mathbb{V} \rightarrow \mathbb{V}$ satisfy (1.4).

The following theorem shows that the (refined) property (2.4) is present under the same conditions as property (1.7).

Theorem 2.4 (Criterion for (2.5)). Assume (1.5) and let $\gamma>0$. Then process (1.2) has property (2.5) if and only if $\gamma$ satisfies condition (2.3).

Proof. 1. Assume (2.3), (1.4) and $0<\Delta t \leq \gamma \cdot \tau_{0}$. We denote by $E_{k}$ the $k \times 1$ matrix with all entries equal to 1 . Note that, since $R=(I-P) S$ and $S E_{l}=E_{m}$, we have $R E_{l}+P E_{m}=E_{m}$, i.e. $\sum_{j=1}^{l} r_{i j}+\sum_{j=1}^{m} p_{i j}=1$.

We rewrite process (1.2), using notations (2.2), in the form

$$
y_{i}=\sum_{j=1}^{l} r_{i j} x_{j}+\sum_{j=1}^{m} p_{i j}\left(y_{j}+\theta \tau_{0} F_{j}\left(y_{j}\right)\right) \quad(1 \leq i \leq m), \quad \theta=\frac{\Delta t}{\gamma \tau_{0}} .
$$

We denote the column vector in $\mathbb{R}^{l}$ with components $\left\|x_{i}\right\|$ by $\left[\left\|x_{i}\right\|\right]$, and we use a similar notation with regard to $y_{i}$ and $F_{i}\left(y_{i}\right)$. Using the convexity property of the functional $\|\cdot\|$, there follows $\left[\left\|y_{i} \mid\right\|\right] \leq R\left[\left\|x_{j} \mid\right\|\right]+P\left[\left\|y_{i}+\theta \tau_{0} F_{i}\left(y_{i}\right)\right\|\right]$. In view of (1.4) we have $P\left[\left\|y_{i}+\theta \tau_{0} F_{i}\left(y_{i}\right)\right\|\right]=P\left[\left\|\theta\left(y_{i}+\tau_{0} F_{i}\left(y_{i}\right)\right)+(1-\theta) y_{i}\right\|\right] \leq P\left[\left\|y_{i}\right\|\right]$, so that

$$
\left[\left\|y_{i} \mid\right\|\right] \leq(I+\gamma T)^{-1} S\left[\| x_{j}||\right]+\left(I-(I+\gamma T)^{-1}\right)\left[\left\|y_{i}\right\|\right],
$$

i.e. $(I+\gamma T)^{-1}\left[\left\|y_{i}\right\|\right] \leq(I+\gamma T)^{-1} S\left[\left\|x_{j}\right\|\right]$. In view of Theorem 2.3, the matrices $S$ and $I+\gamma T$ are nonnegative, so that (2.4) follows. Statement (2.5) has thus been proved.

2. Conversely, assume (2.5). We shall use the notation

$$
\operatorname{sgn}(\alpha)=1(\text { for } \alpha \geq 0), \quad \operatorname{sgn}(\alpha)=-1 \quad(\text { for } \alpha<0) .
$$

Applying (2.5) with $\mathbb{V}=\mathbb{R},\|v\|=v, F_{i}=0, x_{j}=\operatorname{sgn}\left(s_{i_{0} j}\right)$, we see from (2.4) that $\sum_{j}\left|s_{i_{0} j}\right| \leq \sum_{j}\left|s_{i_{0} j}\right| \operatorname{sgn}\left(s_{i_{0} j}\right)$, so that $s_{i_{0} j} \geq 0$. Hence, all $s_{i j} \geq 0$.

For any given $x_{j}, y_{i}$, property (2.4) thus implies (1.6). It follows that (2.5) implies (1.7) and - by Theorem 2.2 - also (2.3) 


\subsection{General bounds with seminorms $\|\cdot\|$}

With an eye to cases where (1.5) or (1.7) (with $\gamma>0$ ) is violated, we shall review and extend, in this section, some results from the literature about bounds which are more general than (1.6) or (2.4). We shall focus on the general bounds

$$
\begin{gathered}
\left\|y_{i}\right\| \leq \mu_{i} \cdot \max _{1 \leq j \leq l}\left\|x_{j}\right\| \quad(\text { for } 1 \leq i \leq m), \\
\left\|y_{i}\right\| \leq \sum_{j=1}^{l} \mu_{i j}\left\|x_{j}\right\| \quad(\text { for } 1 \leq i \leq m),
\end{gathered}
$$

where for the time being $\mu_{i}$ and $\mu_{i j}$ denote arbitrary coefficients. Note that (1.6) or (2.4) can be viewed as special cases of (2.7) and (2.8), respectively.

In this section, we focus on the situation where $\|\cdot\|$ is a seminorm, i.e.

$$
\|v+w\| \leq\|v\|+\|w\| \text { and }\|\lambda v\|=|\lambda|\|v\| \quad \text { (for all real } \lambda \text { and } v, w \in \mathbb{V}) .
$$

We shall say that process (1.2) satisfies the bound (2.7) or (2.8) (for given stepsize $\Delta t$, vector space $\mathbb{V}$, seminorm $\|$.$\| and functions F_{i}: \mathbb{V} \rightarrow \mathbb{V}$ ), if (2.7) or (2.8), respectively, holds whenever $x_{i}$ and $y_{i} \in \mathbb{V}$ satisfy (1.2). The following two statements are obvious variants of (1.7), (2.5), respectively.

Condition $0<\Delta t \leq \gamma \cdot \tau_{0}$ implies that process (1.2) satisfies the bound (2.7), whenever $\mathbb{V}$ is a vector space, $\|\cdot\|$ a seminorm on $\mathbb{V}$, and the functions $F_{i}: \mathbb{V} \rightarrow \mathbb{V}$ satisfy (1.4).

Condition $0<\Delta t \leq \gamma \cdot \tau_{0}$ implies that process (1.2) satisfies the bound (2.8), whenever $\mathbb{V}$ is a vector space, $\|\cdot\|$ a seminorm on $\mathbb{V}$, and the functions $F_{i}: \mathbb{V} \rightarrow \mathbb{V}$ satisfy (1.4).

In formulating conditions on $\gamma$ for (2.10) or (2.11) to be fulfilled, we need some notations. For any matrix $A=\left(a_{i j}\right)$, we define the matrix $|A|$ by $|A|=\left(\left|a_{i j}\right|\right)$. For square matrices $A$, we denote the spectral radius by $\operatorname{spr}(A)$. Furthermore we introduce the $m \times 1$ matrix

$$
\left(\mu_{i}\right)=\left(\mu_{1}, \mu_{2}, \cdots, \mu_{m}\right)^{T}
$$

and the $m \times l$ matrix

$$
\left(\mu_{i j}\right)=\left(\begin{array}{ccc}
\mu_{11} & \cdots & \mu_{1 l} \\
\vdots & & \vdots \\
\mu_{m 1} & \cdots & \mu_{m l}
\end{array}\right) .
$$

We shall relate (2.10) and (2.11), respectively, to the following conditions on $\gamma$ :

$$
\begin{aligned}
& \operatorname{spr}(|P|)<1 \quad \text { and } \quad(I-|P|)^{-1}|R| E_{l} \leq\left(\mu_{i}\right) ; \\
& \operatorname{spr}(|P|)<1 \quad \text { and } \quad(I-|P|)^{-1}|R| \leq\left(\mu_{i j}\right) .
\end{aligned}
$$

The following theorem is a variant of a result given earlier in the literature, see [13]. In fact, when all $\mu_{i}$ are equal to each other, part (I) of the theorem is an immediate corollary to Theorem 2.2 in the paper just mentioned.

Theorem 2.5 (Criteria for (2.10), (2.11)). Consider process (1.2). Let $\gamma>0$ and arbitrary $\mu_{i}, \mu_{i j}$ be given. Then the following two propositions are valid.

(I) Property (2.10) is present, if and only if $\gamma$ is such that condition (2.13) is fulfilled.

(II) Property (2.11) is present, if and only if $\gamma$ is such that condition (2.14) is fulfilled. 
Proof. Conditions (2.13), (2.14) imply (2.10) and (2.11), respectively, by similar arguments as used in part 1 of the proof of Theorem 2.4. Using the arguments of the mentioned proof and (2.9), we get now $\left[\| y_{i}||\right] \leq|R|\left[|| x_{j}||\right]+|P|\left[|| y_{i}||\right]$ instead of (2.6). There follows $\left[\| y_{i}||\right] \leq(I-|P|)^{-1}|R|\left[|| x_{j}||\right]$. By $(2.14)$ we arrive at $(2.11)$. Since $(I-|P|)^{-1}|R|\left[|| x_{j}||\right] \leq$ $\left.(I-|P|)^{-1}|R| E_{l} \cdot \max _{k}|| x_{k}||\right]$, by (2.13) we arrive at (2.10).

The necessity of the conditions (2.13) and (2.14) can be proved by almost the same arguments as already given in [13, Section 4.2].

Theorem 2.5 has a wider scope, certainly, than the theorems of Section 2.2 , in that $\mu_{i}$ and $\mu_{i j}$ are arbitrary coefficients and assumption (1.5) is not needed.

On the other hand, it is in general much more difficult to see whether conditions (2.13), (2.14) are fulfilled than to check criterion (2.3). Moreover, unlike the theorems in Section 2.2, Theorem 2.5 is only relevant to seminorms and not to arbitrary functionals satisfying (1.3). These obvious weaknesses of Theorem 2.5 are among the reasons for dealing in Section 3 with bounds of a very special form.

\section{Bounds of a special form}

\subsection{Special choices for $\mu_{i}, \mu_{i j}$}

Consider the special choices

$$
\mu_{i}=\sum_{j}\left|s_{i j}\right| \quad \text { and } \quad \mu_{i j}=\left|s_{i j}\right| .
$$

Below we shall study the bounds (2.7), (2.8) with these special choices, respectively, i.e.

$$
\begin{gathered}
\left\|y_{i}\right\| \leq\left(\sum_{j=1}^{l}\left|s_{i j}\right|\right) \cdot \max _{1 \leq j \leq l}\left\|x_{j}\right\| \quad(1 \leq i \leq m), \\
\left\|y_{i}\right\| \leq \sum_{j=1}^{l}\left|s_{i j}\right|\left\|x_{j}\right\| \quad(1 \leq i \leq m) .
\end{gathered}
$$

We are led to studying these special bounds by the following three considerations.

First of all, property (2.10) with $\mu_{i}=\sum_{j}\left|s_{i j}\right|$, as well as property (2.11) with $\mu_{i j}=\left|s_{i j}\right|$, can be interpreted as an extension, to all $F_{i}$ satisfying (1.4), of a bound which is trivially fulfilled when $F_{i}(v) \equiv 0$. In fact, in the subsequent Theorem 3.4, we shall see that the bounds (2.7), (2.8) with coefficients (3.1) are best possible in the sense that, for any $\gamma>0$, properties (2.10), (2.11) cannot be valid with coefficients smaller than (3.1).

Secondly, Theorem 3.8 below shows that the equalities $\mu_{i}=\sum_{j}\left|s_{i j}\right|$ are necessary in order that any bound (2.7) holds for a natural class of functionals $\|\cdot\|$ (satisfying (1.3)) that is larger than the class of seminorms. The theorem shows also that the equalities $\mu_{i j}=\left|s_{i j}\right|$ must be fulfilled in order that any bound (2.8) holds for the class of functionals $\|\cdot\|$ just mentioned.

Finally and most importantly in view of applications, the above criteria (2.13) and (2.14), respectively, will turn out to reduce to much simpler forms when $\mu_{i}=\sum_{j}\left|s_{i j}\right|$ or $\mu_{i j}=\left|s_{i j}\right|$.

\subsection{Simplifying (2.13) and (2.14) when $\mu_{i}=\sum_{j}\left|s_{i j}\right|$ and $\mu_{i j}=\left|s_{i j}\right|$}

In this section we shall analyse and simplify the above conditions (2.13), (2.14) in the situation (3.1). Our first result is as follows:

Lemma 3.1 (Conditions (2.13), (2.14) with $\mu_{i}=\sum_{j}\left|s_{i j}\right|$ and $\mu_{i j}=\left|s_{i j}\right|$, respectively). Condition (2.13) with $\mu_{i}=\sum_{j}\left|s_{i j}\right|$ is equivalent to (2.14) with $\mu_{i j}=\left|s_{i j}\right|$. 
Proof. To prove the lemma, we assume $\operatorname{spr}(|P|)<1$ and (3.1).

Suppose (2.13) is fulfilled. Since $\left(\mu_{i}\right)=|S| E_{l}=\left|(I-P)^{-1} R\right| E_{l} \leq(I-|P|)^{-1}|R| E_{l}$, condition (2.13) is equivalent to $|S| E_{l}=(I-|P|)^{-1}|R| E_{l}$, which can be rewritten as $|S| E_{l}=|P||S| E_{l}+|R| E_{l}$. Because of the last equality and $|S|=|R+P S| \leq|R|+|P||S|$, it follows that

$$
|S|=|P||S|+|R| .
$$

Hence, $(I-|P|)^{-1}|R|=|S|=\left(\mu_{i j}\right)$, which implies $(2.14)$.

Conversely, (2.14) implies $(I-|P|)^{-1}|R| E_{l} \leq|S| E_{l}$, i.e. (2.13).

Below, we shall specify situations in which conditions (2.13) and (2.14) with the choice (3.1) can be simplified to one of the subsequent four requirements:

$$
\begin{gathered}
\operatorname{spr}(|P|)<1 \text { and }|P S|=|P||S| \leq|S|, \quad|R| \leq|S| \\
\operatorname{spr}(|P|)<1 \text { and } P S=|P| S, \quad R \geq 0 \\
\operatorname{spr}(P)<1 \text { and } P \geq 0, \quad R \geq 0 \\
P \geq 0, \quad R \geq 0, \quad S \geq 0 .
\end{gathered}
$$

Lemma 3.2 (Simplifications of (2.13) and (2.14), with the choice (3.1)).

(I) Condition (2.13) as well as condition(2.14), with the choice (3.1), is equivalent to (3.4).

(II) If $S \geq 0$ then condition (3.4) is equivalent to (3.5).

(III) Assume $S$ has no row equal to zero. Then the three conditions (3.5), (3.6) and (3.7) are equivalent to each other.

Proof. (I) In view of Lemma 3.1, it is enough to show that (2.14) with $\mu_{i j}=\left|s_{i j}\right|$ is equivalent to (3.4).

From the proof of Lemma 3.1 it is evident that condition (2.14), with $\mu_{i j}=\left|s_{i j}\right|$, is equivalent to

$$
\operatorname{spr}(|P|)<1 \text { and }|S|=|P||S|+|R| .
$$

The last equality implies $|P||S|=|P S|$, because $|S|=|P S+R| \leq|P S|+|R| \leq|P||S|+|R|$. Furthermore, because $S=P S+R$, we have

$$
|S|=|P S|+|R|
$$

as soon as $|P S| \leq|S|$ and $|R| \leq|S|$. It follows that condition (3.8) is equivalent to (3.4).

(II) Assume $S \geq 0$. In order to prove the equivalence of (3.4) and (3.5), assume $\operatorname{spr}(|P|)<1$.

Suppose (3.4) is fulfilled. Since $R=S-P S$ and $|S|=|S-P S|+|P S|$, we have

$$
|R|+|P S|=S=R+P S \leq R+|P S|=R+|P| S,
$$

which implies $R \geq 0$ and $P S=|P| S$. Therefore we have (3.5).

Conversely, from (3.5) and $S=R+P S$ we have

$$
|P||S|+|R|=|S|=|P S+R| \leq|P S|+|R| \leq|P||S|+|R| .
$$

Hence, (3.5) implies (3.4).

(III) Assume $S$ has no row equal to zero. We shall prove successively that (3.5) $\Rightarrow(3.6)$ $\Rightarrow(3.7) \Rightarrow(3.6) \Rightarrow(3.5)$. 
Assume (3.5). Since $(I-|P|) S \geq 0$, we have $S=(I-|P|)^{-1}(I-|P|) S \geq 0$. Denoting by $\sigma_{i}$ the entries of $S E_{l}$, we have $\sigma_{i}=\sum_{j} s_{i j}>0$ (for $\left.1 \leq i \leq m\right)$. Since $(|P|-P) S=0$, we have $(|P|-P) S E_{l}=0$ and thus $\sum_{j}\left(\left|p_{i j}\right|-p_{i j}\right) \sigma_{j}=0$. Hence, $P \geq 0$, and therefore we have (3.6).

Furthermore, (3.6) implies that $S=(I-P)^{-1} R=\left(I+P+P^{2}+\ldots\right) R \geq 0$, so that (3.6) implies (3.7).

In order to prove that property (3.7) leads to (3.6), it is enough to show that $\operatorname{spr}(P)<1$. Introducing $D=\operatorname{Diag}\left(\sigma_{1}, \ldots, \sigma_{m}\right)$ with $\sigma_{i}=\sum_{j} s_{i j}$, we have

$$
D^{-1} P D E_{m}=D^{-1} P S E_{l}=D^{-1}(S-R) E_{l} \leq D^{-1} S E_{l}=E_{m} .
$$

It follows that $\operatorname{spr}(P)=\operatorname{spr}\left(D^{-1} P D\right) \leq 1$. Since $P=I-(I+\gamma T)^{-1} \geq 0$ has no eigenvalue 1 , we conclude from the Perron-Frobenius theory (see e.g. [11, p. 503]) that $\operatorname{spr}(P)<1$.

It is easy to see that (3.6) leads to (3.5).

Remark 3.3. Let $\gamma>0$. Then (3.6) is equivalent to

$$
P \geq 0, \quad R \geq 0, \quad T \geq 0 .
$$

In order to show this, first assume (3.6). Then $I+\gamma T=(I-P)^{-1}=I+P+P^{2}+\ldots \geq I$, which yields (3.9).

Next suppose that (3.9) is fulfilled. Applying the Perron-Frobenius theory as presented e.g. in [11, p. 503], it follows that there is a vector $x \in \mathbb{R}^{m}$ with $0 \leq x \neq 0$, such that $P x=\lambda x$ where $\lambda=\operatorname{spr}(P)$. Clearly, $(I+\gamma T)^{-1} x=(I-P) x=(1-\lambda) x$, and therefore

$$
x=(1-\lambda)(I+\gamma T) x .
$$

Because $T x \geq 0$, the assumption that $\lambda \geq 1$, would lead to:

$$
0 \leq x=(1-\lambda) x+\gamma(1-\lambda) T x \leq(1-\lambda) x \leq 0 .
$$

This would imply $x=0$, which is a contradiction; therefore $\operatorname{spr}(P)<1$.

\subsection{Special bounds with seminorms $\|\cdot\|$}

Clearly, with the choice (3.1), the properties (2.10), (2.11), respectively, reduce to

Condition $0<\Delta t \leq \gamma \cdot \tau_{0}$ implies that process (1.2) satisfies the bound (3.2), whenever $\mathbb{V}$ is a vector space, $\|\cdot\|$ a seminorm on $\mathbb{V}$, and the functions $F_{i}: \mathbb{V} \rightarrow \mathbb{V}$ satisfy (1.4).

Condition $0<\Delta t \leq \gamma \cdot \tau_{0}$ implies that process (1.2) satisfies the bound (3.3), whenever $\mathbb{V}$ is a vector space, $\|\cdot\|$ a seminorm on $\mathbb{V}$, and the functions $F_{i}: \mathbb{V} \rightarrow \mathbb{V}$ satisfy (1.4).

In this section we shall analyse these two properties, and arrive at relatively simple conditions on $\gamma$ for the properties to be present.

But, we present first Theorem 3.4, which shows a crucial feature of (3.10), (3.11): the theorem tells us that the estimates (3.2), (3.3), occurring in (3.10), (3.11), are best possible in that - for any $\gamma>0$ - properties (2.10), (2.11) cannot be valid with smaller choices for $\mu_{i}$ and $\mu_{i j}$ than (3.1). We have

Theorem 3.4 (Lower bounds for $\mu_{i}$ and $\mu_{i j}$ ).

(I) If $\gamma>0$ and $\mu_{i}$ are such that (2.10) holds, then $\mu_{i} \geq \sum_{j}\left|s_{i j}\right|$ (for $\left.1 \leq i \leq m\right)$.

(II) If $\gamma>0$ and $\mu_{i j}$ are such that (2.11) holds, then $\mu_{i j} \geq\left|s_{i j}\right|$ (for $1 \leq i \leq m, 1 \leq j \leq l$ ).

Proof. In order to prove statement (I), assume (2.10) holds with $\gamma>0$ and $\mu_{i_{0}}<\sum_{j}\left|s_{i_{0} j}\right|$ for some index $i_{0}$. Then, in the situation where $\mathbb{V}=\mathbb{R},\|v\|=|v|, F_{i}=0$ and $x_{j}=\operatorname{sgn}\left(s_{i_{0} j}\right)$, we have

$$
\left\|\sum_{j} s_{i_{0} j} x_{j}\right\| \leq \mu_{i_{0}} \cdot \max _{1 \leq j \leq l}\left\|x_{j}\right\|<\sum_{j}\left|s_{i_{0} j}\right|=\left\|\sum_{j} s_{i_{0} j} x_{j}\right\| .
$$


This yields a contradiction, so that (I) must be true.

To prove statement (II), assume (2.11) holds with $\gamma>0$ and $\mu_{i_{0} j_{0}}<\left|s_{i_{0} j_{0}}\right|$ for some pair $\left(i_{0}, j_{0}\right)$. Then, applying (2.11) to the situation where $\mathbb{V}=\mathbb{R},\|v\|=|v|, F_{i}=0$, $x_{j}=\operatorname{sgn}\left(s_{i_{0} j}\right)\left(\right.$ for $\left.j=j_{0}\right)$ and $x_{j}=0\left(\right.$ for $\left.j \neq j_{0}\right)$, we arrive at

$$
\left\|s_{i_{0} j_{0}} x_{j_{0}}\right\| \leq \mu_{i_{0} j_{0}}\left\|x_{j_{0}}\right\|<\left|s_{i_{0} j_{0}}\right|=\left\|s_{i_{0} j_{0}} x_{j_{0}}\right\| .
$$

This yields again a contradiction, so that (II) must be true.

Our main result about properties (3.10), (3.11) will be formulated in Theorem 3.5. The theorem shows that criteria for the properties are possible which are in general much simpler than (2.13) and (2.14).

Theorem 3.5 (Simplified criteria for (3.10) and (3.11)). Consider process (1.2), and let $\gamma>0$. Then the following propositions are valid

(I) Condition (3.4) is necessary and sufficient for property (3.10) as well as for property (3.11).

(II) If $S \geq 0$, then condition (3.5) is necessary and sufficient for property (3.10) as well as for property (3.11).

(III) If $S \geq 0$ has no row equal to zero, then condition (2.3) is necessary and sufficient for property (3.10) as well as for property (3.11).

Proof. Part (I) follows from a combination of Theorem 2.5 and Lemma 3.2.

Part (II) follows from part (I) and Lemma 3.2.

In order to prove statement (III), assume $S \geq 0$ has no row equal to zero. Combining part (II) of Theorem 3.5 and part (III) of Lemma 3.2, it follows that (3.10) as well as (3.11) is equivalent to (3.7). Because $S \geq 0$, condition (3.7) is equivalent to (2.3).

Property (3.11) is a-priori stronger than (3.10). Therefore the essence of the above theorem is that conditions (3.4), (3.5) and (2.3), under the appropriate assumptions on $S$, imply the strong statement (3.11), whereas already the weaker statement (3.10), under the same assumptions on $S$, implies conditions (3.4), (3.5) and (2.3).

\subsection{Special bounds with general sublinear functionals $\|\cdot\|$}

In this section we shall derive conditions for bounds of type (2.7), (2.8) where the functional $\|\cdot\|$ is not necessarily a seminorm. The following two examples provide some motivation for dealing with such bounds.

Example 3.6. Consider the functionals $\|v\|=\|v\|_{+}$and $\|v\|=\|v\|_{-}$defined by

$$
\|v\|_{+}=\max _{i} v_{i}, \quad\|v\|_{-}=-\min _{i} v_{i} \quad\left(\text { for } v=\left(v_{1}, v_{2}, \ldots, v_{M}\right)^{T} \in \mathbb{V}=\mathbb{R}^{M}\right) .
$$

These two functionals are no seminorms. But, they are highly relevant to discrete maximum principles for actual numerical processes, cf. [17, p. 118], [22, p. 1235].

Example 3.7. Another useful functional, violating (2.9), is given by

$$
\|v\|_{0}=-\min \left\{0, v_{1}, \ldots, v_{M}\right\} \quad\left(\text { for } v=\left(v_{1}, v_{2}, \ldots, v_{M}\right)^{T} \in \mathbb{V}=\mathbb{R}^{M}\right) .
$$

For this non-negative functional we have $\|v\|_{0}=0$ if and only if $v \geq 0$, where this inequality is to be interpreted component-wise. One sees that any boundedness property $\max _{i}\left\|y_{i}\right\|_{0} \leq$ $\mu \cdot \max _{j}\left\|x_{j}\right\|_{0}$ implies the preservation-of-nonnegativity property: $y_{i} \geq 0$ (for $1 \leq i \leq m$ ) whenever all $x_{j} \geq 0$. For the practical relevance of this property, e.g. in the numerical solution of reaction-diffusion-convection equations, one may consult e.g. [17]. 
Since the functionals in (3.12) and (3.13) violate condition (2.9), the material of Sections 2.3 and 3.3 does not apply. It is therefore natural to look for versions of Theorems 2.5 , 3.4 and 3.5 which are relevant to classes of functionals which are larger than the one specified by (2.9). Below we shall focus on functionals $\|\cdot\|$ which are only required to be sublinear, i.e.

$$
\|\alpha v+\beta w\| \leq \alpha\|v\|+\beta\|w\| \quad \text { (for all } \alpha, \beta \geq 0 \text { and } v, w \in \mathbb{V})
$$

Note that (3.14) is equivalent to $\|v+w\| \leq\|v\|+\|w\|,\|\lambda v\|=\lambda\|v\|$ (for all $\lambda \geq 0$ and $v, w \in \mathbb{V})$. The functionals in (3.12) and (3.13) satisfy (3.14).

In line with the above, we shall study the question for which values $\gamma>0$ process (1.2) has either of the following two properties:

Condition $0<\Delta t \leq \gamma \cdot \tau_{0}$ implies the bound (2.7), whenever $\mathbb{V}$ is a vector space, $\|\cdot\|$ a sublinear functional on $\mathbb{V}$, and the functions $F_{i}: \mathbb{V} \rightarrow \mathbb{V}$ satisfy (1.4).

Condition $0<\Delta t \leq \gamma \cdot \tau_{0}$ implies the bound (2.8), whenever $\mathbb{V}$ is a vector space, $\|\cdot\|$ a sublinear functional on $\mathbb{V}$, and the functions $F_{i}: \mathbb{V} \rightarrow \mathbb{V}$ satisfy (1.4).

The following theorem may be viewed as a variant of Theorem 3.4 tuned to sublinear functionals. It shows, somewhat surprisingly, that we loose nothing by focusing on bounds with the coefficients (3.1).

Theorem 3.8 (Expressions for $\mu_{i}$ and $\mu_{i j}$ ).

(I) If $\gamma>0$ and $\mu_{i}$ are such that (3.15) holds, then $\mu_{i}=\sum_{j}\left|s_{i j}\right|(1 \leq i \leq m)$ and $S \geq 0$.

(II) If $\gamma>0$ and $\mu_{i j}$ are such that (3.16) holds, then $\mu_{i j}=\left|s_{i j}\right|(1 \leq i \leq m, 1 \leq j \leq l)$ and $S \geq 0$.

Proof. (I) It follows from Theorem 3.4 that

$$
\sum_{j}\left|s_{i j}\right| \leq \mu_{i} \quad(\text { for } 1 \leq i \leq m) .
$$

Applying (3.15) to the situation where $\mathbb{V}=\mathbb{R},\|v\|=v, F_{i}(v) \equiv 0$, and choosing successively all $x_{j}=1$ and all $x_{j}=-1$, we find $\sum_{j} s_{i j} \leq \mu_{i}$ and $\left(-\sum_{j} s_{i j}\right) \leq\left(-\mu_{i}\right)$, respectively. Hence

$$
\mu_{i}=\sum_{j} s_{i j}(\text { for } 1 \leq i \leq m) .
$$

Combining (3.17) and (3.18), we arrive at proposition (I).

(II) It follows from Theorem 3.4 that

$$
\sum_{j}\left|s_{i j}\right| \leq \sum_{j} \mu_{i j} \quad(\text { for } 1 \leq i \leq m)
$$

Applying (3.16) to the situation where $\mathbb{V}=\mathbb{R},\|v\|=v, F_{i}(v) \equiv 0$, we conclude that $\sum_{j} s_{i j} x_{j}=y_{i} \leq \sum_{j} \mu_{i j} x_{j}(1 \leq i \leq m)$, for all real values $x_{j}$. This implies

$$
\mu_{i j}=s_{i j}(\text { for } 1 \leq i \leq m, 1 \leq j \leq l) .
$$

Combining (3.19) and (3.20), we arrive at proposition (II).

Theorem 3.8 shows that the bounds (3.2), (3.3), respectively, are the only bounds of type (2.7), (2.8) which make sense in the context of general sublinear functionals $\|\cdot\|$. Accordingly, we shall focus on the following variants of (3.15) and (3.16), respectively:

Condition $0<\Delta t \leq \gamma \cdot \tau_{0}$ implies that process (1.2) satisfies the bound (3.2), whenever $\mathbb{V}$ is a vector space, $\|\cdot\|$ a sublinear functional on $\mathbb{V}$, and the functions $F_{i}: \mathbb{V} \rightarrow \mathbb{V}$ satisfy (1.4), 
Condition $0<\Delta t \leq \gamma \cdot \tau_{0}$ implies that process (1.2) satisfies the bound (3.3), whenever $\mathbb{V}$ is a vector space, $\|\cdot\|$ a sublinear functional on $\mathbb{V}$, and the functions $F_{i}: \mathbb{V} \rightarrow \mathbb{V}$ satisfy (1.4).

Our main result about properties (3.21), (3.22) has been formulated in Theorem 3.9. The theorem can be regarded as a neat version of Theorem 3.5, parts (I) and (III), adapted to sublinear functionals.

Theorem 3.9 (Criteria for (3.21) and (3.22)). Consider process (1.2), and let $\gamma>0$. Then the following propositions are valid

(I) Condition (3.6) is necessary and sufficient for property (3.21) as well as for property (3.22).

(II) If $S \geq 0$ has no row equal to zero, then condition (2.3) is necessary and sufficient for property (3.21) as well as for property (3.22).

Proof. (I) We prove necessity and sufficiency of (3.6) separately.

1 (Sufficiency). It is easy to see that property (3.22) implies (3.21). Therefore, it is enough to prove that condition (3.6) implies (3.22). The last implication can be proved by almost the same arguments as used in part 1 of the proof of Theorem 2.4 in Section 2 . Note that again the inequalities $I+\gamma T \geq 0$ and $S \geq 0$ are needed, which follow now from: $I+\gamma T=(I-P)^{-1}=I+P+P^{2}+\ldots \geq 0$ and $S=(I-P)^{-1} R \geq 0$.

2 (Necessity). For proving the necessity it is enough to show that: (3.21) implies (3.6). To prove this implication, we (only) assume (3.21) to hold in the situation where

$$
\mathbb{V}=\mathbb{R}^{m}, \quad\|v\|=\max _{k} v^{[k]} \text { (for } v \in \mathbb{V} \text { with components } v^{[k]}(1 \leq k \leq m) \text { ). }
$$

We define functions $F_{j}: \mathbb{V} \rightarrow \mathbb{V}$ by

$$
F_{j}(v)=\tau_{0}^{-1}\left(-y_{j}+z_{j}\right) \quad\left(\text { for } v=y_{j}\right), \quad F_{j}(v)=0 \quad \text { (otherwise), }
$$

where $y_{j}, z_{j}$ are vectors in $\mathbb{V}$ - to be specified below - satisfying

$$
\left.\left\|z_{j}\right\| \leq\left\|y_{j}\right\| \quad(1 \leq j \leq m)\right)
$$

Clearly the functions $F_{j}$ defined in this fashion satisfy (1.4).

We consider the matrices $P=\left(p_{i j}\right), R=\left(r_{i j}\right)$ (cf. (2.2)) and define the components of $x_{j}, z_{j} \in \mathbb{V}$ by $x_{j}^{[k]}=-1 \quad$ (if $r_{k j}<0$ ), $x_{j}^{[k]}=0$ (otherwise), and $z_{j}^{[k]}=-1 \quad$ (if $p_{k j}<0$ ), $z_{j}^{[k]}=0$ (otherwise). We define the vectors $y_{i} \in \mathbb{V}$ by $y_{i}=\sum_{j=1}^{l} r_{i j} x_{j}+\sum_{j=1}^{m} p_{i j} z_{j} \quad(1 \leq$ $i \leq m)$. A short calculation shows that $x_{i}, y_{i}$ satisfy (1.2) with the functions $F_{j}$ as defined above and $\Delta t=\gamma \tau_{0}$.

We denote by $\rho_{i}$ the sum of the absolute values of the negative entries in the $i$-th row of $R$, and by $\pi_{i}$ the sum of the absolute values of the negative entries in the $i$-th row of $P$. By the definition of $y_{i}$, we have $\left\|y_{i}\right\| \geq y_{i}^{[i]}=\rho_{i}+\pi_{i}(1 \leq i \leq m)$. Because $\left\|z_{i}\right\| \leq 0$, the inequalities (3.23) are in force, so that (1.4) is valid.

Applying (3.21) to the situation at hand, there follows

$$
\rho_{i}+\pi_{i} \leq\left\|y_{i}\right\| \leq\left(\sum_{j}\left|s_{i j}\right|\right) \cdot \max _{j}\left\|x_{j}\right\| \leq 0 \quad(1 \leq i \leq m),
$$

which proves $P \geq 0, R \geq 0$. The remaining inequality, $\operatorname{spr}(P)<1$, follows e.g. by applying Theorem 3.5, part (I).

(II) Let condition (2.3) be fulfilled. Then (3.7) holds as well. So, by Lemma 3.2, part(III), condition (3.6) is fulfilled. From part (I) (of Theorem 3.9) we conclude that (3.21) and (3.22) hold.

Conversely, assume (3.21) or (3.22). By Theorem 3.5, part(III), we arrive at (2.3).

Since property (3.22) is a-priori stronger than (3.21), the essence of the above theorem is that conditions (3.6), (2.3) (under the appropriate assumptions on $S$ ) imply the strong statement (3.22), whereas already the weaker statement (3.21) implies (3.6) and (2.3)(under the same assumptions on $S$ ). 


\subsection{Various natural questions}

In this section we ask and answer five natural questions about possible simplifications or extensions of Lemma 3.2 and Theorems 3.5, 3.9. For each of these questions we will provide counterexamples.

Question 3.10. Because (3.5), (3.6), (3.7) and (2.3) are more simple in appearance than (3.4), the question arises of whether condition (3.4) can be replaced by one of these four conditions in Lemma 3.2 (part (I)) or in Theorem 3.5 (part(I)).

To answer this question, consider (1.2) with $l=2, m=1$ and $s_{11}=-2, s_{12}=1$, $t_{11}=1$. Let $\gamma>0$. It is easy to see that condition (3.4) is fulfilled. Hence, (3.10) and (3.11) are valid. But, we do not have $R \geq 0$, so that (3.5), (3.6), (3.7) and (2.3) are violated. Therefore, none of the last four conditions can replace condition (3.4) in Lemma 3.2 (part (I)) or in Theorem $3.5(\operatorname{part}(\mathrm{I}))$.

Question 3.11. Because (3.6), (3.7) and (2.3) are more simple conditions than (3.5), the question arises of whether condition (3.5) can be replaced by one of these three conditions in Lemma 3.2 (part (II)) or in Theorem 3.5 (part(II)).

The following counterexample proves that such replacement is not possible. Consider process (1.2) with $l=m=1$ and $s_{11}=0, t_{11}=-1$. Let $\gamma=0.25$. One easily sees that condition (3.5) is fulfilled, so that (3.10) and (3.11) are valid. But, we do not have $P \geq 0$, so that $(3.6),(3.7)$ and $(2.3)$ are violated. Therefore, none of the last three conditions can replace condition (3.5) in Lemma 3.2 (part (II)) or in Theorem 3.5 (part(II)).

Question 3.12. Because (2.3) is more simple a condition than (3.6), the question arises of whether condition (3.6) can be replaced by (2.3) in Theorem 3.9 (part(I)).

The following counterexample proves that such replacement is not possible. Consider process (1.2) with $l=m=1$ and $s_{11}=0, t_{11}=-1$. Let $\gamma=2$. One easily sees that condition (3.6) is violated, so that (3.21) and (3.22) are not valid. But (2.3) is fulfilled. Therefore, condition (2.3) cannot replace (3.6) in Theorem 3.9 (part(I)).

Question 3.13. One may ask whether the condition $S \geq 0$ can be omitted in Theorem 3.5 (part(III)) or in Theorem $3.9(\operatorname{part}(\mathrm{II}))$.

To answer this question, consider (1.2) with $l=m=1$ and $s_{11}=-1, t_{11}=-1$. Let $\gamma=2$. It is easy to see that we have condition (2.3) but not (3.4) or (3.6). Hence, (3.10), (3.11), (3.21), (3.22) are not valid but (2.3) holds. Therefore, the condition $S \geq 0$ cannot be omitted in Theorem 3.5 (part(III)) or in Theorem 3.9 (part(II)).

Question 3.14. Finally, we consider the question of whether the condition of $S$ having no row equal to zero, can be omitted in Theorem 3.9 (part(II)). A negative answer to this question easily follows from the counterexample used above in resolving Question 3.12.

\section{Applications of the theory}

\subsection{Preliminaries}

Below we shall illustrate the preceding theory by applying it to some well-known numerical methods. In these applications, we will restrict ourselves, for ease of representation, to autonomous problems, i.e. $F$ in (1.1) is independent of $t$, and $F_{j}=F$ in (1.2). Condition (1.4) thus reduces to

$$
\left\|v+\tau_{0} F(v)\right\| \leq\|v\| \quad(\text { for all } v \in \mathbb{V}) .
$$

In Section 4.2 we shall deal with the two-step $(k=2)$ Adams-Bashforth LMM and in Section 4.3 with a class of $k$-step 2 -stage methods. All of these methods generate vectors $u_{n} \in \mathbb{V}$ (for $n \geq k$ ) from starting vectors $u_{0}, \ldots, u_{k-1} \in \mathbb{V}$, where $u_{n} \approx u(n \cdot \Delta t)$ and $k$ is 
fixed. We call a $k$-step method bounded with factor $\mu$ (for given stepsize $\Delta t$, vector space $\mathbb{V}$, functional $\|\cdot\|$ and function $F$ ) if

$$
\left\|u_{n}\right\| \leq \mu \cdot \max _{0 \leq j \leq k-1}\left\|u_{j}\right\| \quad(k \leq n \leq k-1+N),
$$

whenever $N \geq 1$ and $u_{n} \in \mathbb{V}(k \leq n \leq k-1+N)$ are generated from any $u_{0}, \ldots, u_{k-1} \in \mathbb{V}$ by $N$ successive applications of the method. Boundedness with factor $\mu=1$ will be referred to as monotonicity of the method.

We recall that boundedness and monotonicity with the so-called total-variation-seminorm (defined by $\|x\|=\|x\|_{T V}=\sum_{i}\left|\xi_{i+1}-\xi_{i}\right|$ for vectors $x$ with components $\xi_{i}$ ) correspond to the important concepts total-variation-bounded and total-variation-diminishing, respectively, cf. e.g. [17, 18].

In the following we shall focus on the situation where the functional $\|\cdot\|$ is a seminorm. We shall consider stepsize-coefficients $\gamma>0$ and factors $\mu$ such that

$$
\begin{aligned}
& \text { Condition } 0<\Delta t \leq \gamma \cdot \tau_{0} \text { implies boundedness with factor } \mu \text {, whenever } \mathbb{V} \text { is } \\
& \text { a vector space with seminorm }\|\cdot\| \text {, and } F: \mathbb{V} \rightarrow \mathbb{V} \text { satisfies (4.1). }
\end{aligned}
$$

In case $\gamma, \mu$ satisfy (4.3), we will say that $\gamma$ is a stepsize-coefficient for boundedness of the method with factor $\mu$; in case $\gamma$ satisfies (4.3) with $\mu=1$, we will call it a stepsizecoefficient for monotonicity. Below we shall look for stepsize-coefficients with property (4.3) by considering representations (1.2) of $N$ consecutive steps of the method under consideration.

\subsection{The two-step Adams-Bashforth method}

The well-known 2-step Adams-Bashforth method reads

$$
u_{n}=u_{n-1}+\Delta t\left[\frac{3}{2} F\left(u_{n-1}\right)-\frac{1}{2} F\left(u_{n-2}\right)\right] ;
$$

it yields approximations $u_{n} \approx u(n \Delta t)(n=2,3, \ldots)$, starting from $u_{0}$ and $u_{1} \approx u(\Delta t)$. In this section we shall look at the relevance of Theorems $2.2,2.4,3.5,3.9$ in the analysis of this method, thereby representing $N$ consecutive steps of (4.4) in two different ways as a process of type (1.2).

In order to describe our first, and most natural, representation of (4.4) in the form (1.2), we put $l=2, m=N+2$, and $x_{1}=u_{0}, x_{2}=u_{1}, y_{i}=u_{i-1}(1 \leq i \leq m)$. Clearly, (4.4) holds for $2 \leq n \leq N+1$ if and only if

$$
\begin{aligned}
& y_{1}=x_{1}, \\
& y_{2}=x_{2}, \\
& y_{i}=x_{2}-\frac{1}{2} \Delta t F\left(y_{1}\right)+\Delta t \sum_{j=2}^{i-2} F\left(y_{j}\right)+\frac{3}{2} \Delta t F\left(y_{i-1}\right) \quad(3 \leq i \leq m) .
\end{aligned}
$$

These relations are the same as (1.2) with $m \times 2$ coefficient matrix $S=\left(s_{i j}\right)$ and $m \times m$ coefficient matrix $T=\left(t_{i j}\right)$ defined by:

$$
S=\left(\begin{array}{cc}
1 & 0 \\
0 & 1 \\
\vdots & \vdots \\
0 & 1
\end{array}\right), \quad T=\left(\begin{array}{cccccc}
0 & & & & & \\
0 & 0 & & & & \\
-\frac{1}{2} & \frac{3}{2} & 0 & & & \\
-\frac{1}{2} & 1 & \frac{3}{2} & 0 & & \\
\vdots & \vdots & \ddots & \ddots & \ddots & \\
-\frac{1}{2} & 1 & \cdots & 1 & \frac{3}{2} & 0
\end{array}\right)
$$

With these definitions, the relations (4.4) (for $2 \leq n \leq N+1$ ) thus hold if and only if (1.2) is fulfilled.

For the matrix $T$ at hand, we have (2.1) for all $\gamma>0$. Furthermore, because (1.5) is fulfilled, one might hope to be able to prove (1.7) or (2.5), for some $\gamma>0$, by applying 
Theorem 2.2 or 2.4 , respectively. If this were possible, such a $\gamma$ would be a stepsizecoefficient for monotonicity in the sense specified in Section 4.1.

However, a short calculation shows that the matrix $P=(I+\gamma T)^{-1}(\gamma T)$ has a negative entry (for any $\gamma>0$ and all $N \geq 1$ ), so that we cannot conclude, by applying Theorem 2.2 or 2.4, that there is $\gamma>0$ for which (1.7) or (2.5) holds. Similarly, Theorems 3.5, 3.9 cannot be applied here so as to arrive at (4.3) with positive $\gamma$. In fact, the following statement can be proved, e.g. by applying the material in [21, Theorem 3.3].

Statement 4.1. For method (4.4) there exists no positive stepsize-coefficient for monotonicity.

In spite of this statement, we shall see below that a positive stepsize-coefficient for boundedness can be determined by applying Theorem 3.5 and representing (4.4) (for $2 \leq$ $n \leq N+1)$ in the form (1.2) with less obvious matrices $S, T$ than used above.

We consider the representation in the form (1.2), with $l=2, m=N, y_{i}=u_{i+1}$ $(1 \leq i \leq m)$ and input vectors

$$
x_{1}=u_{1}+\frac{3}{2} \Delta t F\left(u_{1}\right)-\frac{1}{2} \Delta t F\left(u_{0}\right), \quad x_{2}=-\frac{1}{2} \Delta t F\left(u_{1}\right) .
$$

Clearly, (4.4) $(2 \leq n \leq N+1)$ amounts to

$$
\begin{aligned}
& y_{1}=x_{1}, \\
& y_{i}=x_{1}+x_{2}+\Delta t \sum_{j=1}^{i-2} F\left(y_{j}\right)+\frac{3}{2} \Delta t F\left(y_{i-1}\right) \quad(2 \leq i \leq m) .
\end{aligned}
$$

$N$ steps of (4.4) can thus be represented by (1.2), with $l=2, m=N$ and

$$
S=\left(\begin{array}{cc}
1 & 0 \\
1 & 1 \\
\vdots & \vdots \\
1 & 1
\end{array}\right), \quad T=\left(\begin{array}{ccccc}
0 & & & & \\
\frac{3}{2} & 0 & & & \\
1 & \frac{3}{2} & 0 & & \\
\vdots & \ddots & \ddots & \ddots & \\
1 & \cdots & 1 & \frac{3}{2} & 0
\end{array}\right)
$$

Note that this matrix $S$ violates (1.5), so that the monotonicity theory of Section 2.2 is not relevant here. But, the special boundedness theory of Section 3 still applies.

To be able to apply Theorem 3.5, we shall determine expressions for $P$ and $R$ corresponding to (4.8). A short calculation shows that

$$
(I+\gamma T)^{-1}=\left(\begin{array}{cccc}
q_{0} & & & \\
q_{1} & q_{0} & & \\
\vdots & \ddots & \ddots & \\
q_{m-1} & \cdots & q_{1} & q_{0}
\end{array}\right)
$$

where $q_{0}=1, q_{1}=-\frac{3}{2} \gamma$ and $q_{i}=\left(1-\frac{3}{2} \gamma\right) q_{i-1}+\frac{1}{2} \gamma q_{i-2}$ for $i \geq 2$. It follows that

$$
R=\left(\begin{array}{cc}
r_{0} & 0 \\
r_{1} & r_{0} \\
\vdots & \vdots \\
r_{m-1} & r_{m-2}
\end{array}\right), \quad P=-\left(\begin{array}{cccc}
0 & & & \\
q_{1} & 0 & & \\
\vdots & \ddots & \ddots & \\
q_{m-1} & \cdots & q_{1} & 0
\end{array}\right) \text {, }
$$

where $r_{i}=q_{0}+q_{1}+\cdots+q_{i}$. Using the recurrence relation satisfied by $q_{i}$, one finds for $0<\gamma \leq \frac{4}{9}$ and $i \geq 1$ that $q_{i} \leq 0$ and $\gamma \cdot r_{i}=-\left[(1-\gamma) q_{i}+\frac{\gamma}{2} q_{i-1}\right] \geq 0$. Hence, (2.3) holds for any $\gamma \in\left(0, \frac{4}{9}\right]$. In the rest of this section we assume $\gamma=\frac{4}{9}$.

From proposition (III) of Theorem 3.5, we conclude that process (1.2) has property (3.11). Using this property and (4.6), it follows that condition $0<\Delta t \leq \gamma \cdot \tau_{0}$ implies:

$$
\left\|u_{n}\right\| \leq\left\|u_{1}+\frac{3}{2} \Delta t F\left(u_{1}\right)-\frac{1}{2} \Delta t F\left(u_{0}\right)\right\|+\left\|-\frac{1}{2} \Delta t F\left(u_{1}\right)\right\| \quad(2 \leq n \leq N+1),
$$


whenever $u_{n}$ is generated by applying (4.4) under assumption (4.1), where $\|\cdot\|$ is an arbitrary seminorm on the vector space $\mathbb{V}$.

For $0<\Delta t \leq \gamma \cdot \tau_{0}$ and any seminorm $\|\cdot\|$, we have

$$
\|\Delta t F(v)\|=\left(\Delta t / \tau_{0}\right)\left\|-v+\left(v+\tau_{0} F(v)\right)\right\| \leq 2 \gamma\|v\|,
$$

which can be seen to imply

$$
\left\|u_{1}+\frac{3}{2} \Delta t F\left(u_{1}\right)-\frac{1}{2} \Delta t F\left(u_{0}\right)\right\| \leq\left\|u_{1}\right\|+\gamma\left\|u_{0}\right\| ;
$$

hence,

$$
\left\|u_{1}+\frac{3}{2} \Delta t F\left(u_{1}\right)-\frac{1}{2} \Delta t F\left(u_{0}\right)\right\|+\left\|-\frac{1}{2} \Delta t F\left(u_{1}\right)\right\| \leq(1+\gamma)\left\|u_{1}\right\|+\gamma\left\|u_{0}\right\| .
$$

Combining (4.9) and (4.10) we arrive at the following:

Statement 4.2. The value $\gamma=4 / 9$ is a stepsize-coefficient for boundedness of (4.4) with factor $\mu=17 / 9$.

By applying Theorem 3.9, instead of Theorem 3.5, we find similarly as above that (4.9) holds, whenever $u_{n}(2 \leq n \leq N+1)$ is generated by applying (4.4) under assumption (4.1), where $\|\cdot\|$ is now an arbitrary sublinear functional on the vector space $\mathbb{V}$. But, in the general situation of sublinear functionals, we cannot derive similarly as above that (4.10) is valid.

To give a simple illustration of (4.9), with a sublinear functional $\|\cdot\|$ which is no seminorm, we consider $\mathbb{V}=\mathbb{R}^{M}$ with functional (3.13). Applying Theorem 3.9 to the situation at hand, and defining $v \geq 0$ by nonnegativity of all components of $v \in \mathbb{V}$, yields:

Statement 4.3. Consider $\mathbb{V}=\mathbb{R}^{M}$ with functional $\|\cdot\|=\|\cdot\|_{0}$, (3.13), and assume $F: \mathbb{V} \rightarrow \mathbb{V}$ satisfies (4.1). Then condition $0<\Delta t \leq \frac{4}{9} \tau_{0}$ implies

$$
u_{n} \geq 0 \quad(2 \leq n \leq N+1),
$$

whenever $u_{n}$ is obtained via (4.4) from $u_{0}, u_{1}$ with $u_{1}+\frac{3}{2} \Delta t F\left(u_{1}\right) \geq \frac{1}{2} \Delta t F\left(u_{0}\right), F\left(u_{1}\right) \leq 0$.

We note that, for method (4.4) and any $\gamma>0$, property $u_{n} \geq 0(2 \leq n \leq N+1)$ cannot be proved for $0<\Delta t \leq \gamma \cdot \tau_{0}$, under the more natural assumption that

$$
u_{0} \geq 0, u_{1} \geq 0 \quad \text { and } \quad v+\tau_{0} F(v) \geq 0 \quad \text { (for all } v \in \mathbb{R}^{M} \text { with } v \geq 0 \text { ). }
$$

This can be seen, for example, by considering $\mathbb{V}=\mathbb{R}, F(v) \equiv v$ and $u_{0}=1, u_{1}=0$.

\subsection{Predictor-corrector methods and hybrid multistep methods}

\subsubsection{Notations}

Using an explicit linear multistep method (LMM), with coefficients $\hat{a}_{j}, \hat{b}_{j}$, as a predictor for an implicit LMM, with coefficients $a_{j}, b_{j}$, results in a numerical process of type

$$
\begin{aligned}
& v_{n}=\sum_{j=1}^{k} \hat{a}_{j} u_{n-j}+\Delta t \sum_{j=1}^{k} \hat{b}_{j} F\left(u_{n-j}\right), \\
& u_{n}=\sum_{j=1}^{k} a_{j} u_{n-j}+\Delta t \sum_{j=1}^{k} b_{j} F\left(u_{n-j}\right)+\Delta t b_{0} F\left(v_{n}\right),
\end{aligned}
$$

where $k \geq 1$ is fixed and $n=k, k+1, \ldots$, cf. e.g. [3, 8, 12]. The starting values for this method are $u_{0}, u_{1}, \ldots, u_{k-1} \in \mathbb{V}$. 
Throughout this section we assume $b_{0}>0, \sum_{j=1}^{k} \hat{a}_{j}=1, \sum_{j=1}^{k} a_{j}=1$, as well as zero-stability, i.e. all roots of the equation $\xi^{k}=\sum_{j=1}^{k} a_{j} \xi^{k-j}$ have a modulus $|\xi| \leq 1$, and the roots with $|\xi|=1$ are simple.

Methods of type (4.11) are called predictor-corrector methods if $u_{n}$ and $v_{n}$, respectively, are final and tentative approximations to the solution at $t_{n}=n \Delta t$. If a predictor (4.11a) corresponds to a method with order of accuracy $k$, and a corrector $(4.11 \mathrm{~b})$ to a method with order $k+1$, then the predictor-corrector method (4.11) has order $k+1$. The most popular schemes of this type are obtained by combining the explicit Adams-Bashforth and implicit Adams-Moulton methods, cf. the literature mentioned above.

The formulas (4.11) can also stand for so-called hybrid multistep methods, also known as modified linear multistep methods, where $v_{n}$ approximates the solution at a point $\bar{t}_{n}=$ $(n-\kappa) \Delta t$, with an extra parameter $\kappa \neq 0$; cf. the above literature.

We shall represent $N \geq 1$ steps of the general method (4.11) as a process of type (1.2), were $y=\left[y_{i}\right] \in \mathbb{V}^{m}, m=2 N$, with

$$
y_{i}=u_{k-1+i}, \quad y_{N+i}=v_{k-1+i} \quad \text { for } 1 \leq i \leq N .
$$

For the input vector we take $x=\left[x_{j}\right] \in \mathbb{V}^{l}, l=2 k$, defined by

$$
\begin{array}{rlr}
x_{i}=\sum_{j=i}^{k} a_{j} u_{k-1+i-j}+\Delta t \sum_{j=i}^{k} b_{j} F\left(u_{k-1+i-j}\right) & (1 \leq i \leq k), \\
x_{i+k}=\sum_{j=i}^{k} \hat{a}_{j} u_{k-1+i-j}+\Delta t \sum_{j=i}^{k} \hat{b}_{j} F\left(u_{k-1+i-j}\right) & (1 \leq i \leq k) .
\end{array}
$$

To write the relations $(4.11),(4.12)$ specifying $y_{1}, y_{2}, \ldots, y_{m}$ in a compact way, we give the following definitions. For any $m \times r$ matrix $S=\left(s_{i j}\right)$ we denote by the boldface symbol $\boldsymbol{S}$ the corresponding linear map from $\mathbb{V}^{r}$ to $\mathbb{V}^{m}$, that is, $y=\boldsymbol{S} x$ if $y_{i}=\sum_{j=1}^{r} s_{i j} x_{j} \in \mathbb{V}$ $(1 \leq i \leq m)$. Let $I$ be the $N \times N$ identity matrix. Let $J_{0} \in \mathbb{R}^{N \times k}$ be the matrix that consists of either the first $N$ rows of the $k \times k$ identity matrix (when $1 \leq N<k$ ), or the first $k$ columns of $I$ (when $N \geq k$ ). Furthermore, let $A_{0} \in \mathbb{R}^{N \times N}$ be the lower triangular Toeplitz matrix with diagonal entries 0 , entries $a_{j}$ on the $j$-th lower diagonal $(1 \leq j \leq \min \{k, N-1\})$ and with the remaining entries 0 again. The matrices $B_{0}, \hat{A}_{0}, \hat{B}_{0} \in \mathbb{R}^{N \times N}$ are defined likewise with coefficients $b_{j}, \hat{a}_{j}, \hat{b}_{j}(1 \leq j \leq \min \{k, N-1\})$, respectively (the coefficient $b_{0}$ does not enter into the matrix $B_{0}$ ).

It is easy to see that the relations (4.11) (for $k \leq n \leq k-1+N$ ) are equivalent to

$$
y=\boldsymbol{J} x+\boldsymbol{A} y+\Delta t \boldsymbol{B} \boldsymbol{F}(y),
$$

where $\boldsymbol{F}(y)=\left[F\left(y_{j}\right)\right] \in \mathbb{V}^{m}$, and $J \in \mathbb{R}^{m \times l}, A, B \in \mathbb{R}^{m \times m}$ are given by

$$
J=\left(\begin{array}{cc}
J_{0} & 0 \\
0 & J_{0}
\end{array}\right), \quad A=\left(\begin{array}{cc}
A_{0} & O \\
\hat{A}_{0} & O
\end{array}\right), \quad B=\left(\begin{array}{cc}
B_{0} & b_{0} I \\
\hat{B}_{0} & O
\end{array}\right) .
$$

The generic form (1.2) is thus obtained with coefficient matrices $\left(s_{i j}\right)=S=(I-A)^{-1} J$ and $\left(t_{i j}\right)=T=(I-A)^{-1} B$.

\subsubsection{Monotonicity for (4.11)}

Let us first take a brief look at standard monotonicity with respect to the starting vectors $u_{0}, \ldots, u_{k-1}$. For this, it is convenient to introduce $\check{a}_{j}=a_{j}-\gamma b_{0} \hat{a}_{j}$ and $\check{b}_{j}=b_{j}-\gamma b_{0} \hat{b}_{j}$ (for $j=1, \ldots, k$ ). The relations (4.11) imply that

$$
u_{n}=\sum_{j=1}^{k} \check{a}_{j} u_{n-j}+\Delta t \sum_{j=1}^{k} \check{b}_{j} F\left(u_{n-j}\right)+\gamma b_{0}\left(v_{n}+\frac{\Delta t}{\gamma} F\left(v_{n}\right)\right) .
$$


By combining this equality with (4.11a), we arrive at the following theorem; see also e.g. $[6,22,12]$.

Theorem 4.4. Assume (4.11) holds for $n=k, k+1, \ldots, k-1+N$. Assume (1.3), (1.4), and let $\gamma>0$ be such that

$$
\hat{a}_{j} \geq \gamma \hat{b}_{j} \geq 0, \quad \check{a}_{j} \geq \gamma \check{b}_{j} \geq 0 \quad(j=1, \ldots, k) .
$$

Then the stepsize restriction $0<\Delta t \leq \gamma \cdot \tau_{0}$ implies that

$$
\left\|u_{n}\right\| \leq \max _{0 \leq j \leq k-1}\left\|u_{j}\right\| \quad(k \leq n \leq k-1+N) .
$$

Note that, under a weak irreducibility assumption, condition (4.16) is not only sufficient but also necessary for (4.17), see [22].

However, the methods (4.11) satisfying (4.16) form a small class, excluding popular schemes, for instance obtained by combining explicit and implicit Adams-type methods as indicated above. Furthermore, in view of results for LMMs of [19], one can expect that the stepsize requirement $\Delta t \leq \gamma \cdot \tau_{0}$ (with $\gamma$ such that (4.16) holds) may be unnecessarily restrictive if $\gamma$ is only required to be a stepsize-coefficient for boundedness (in the sense of Section 4.1).

Below we apply the theory of Section 3 in an analysis of (4.11) which is also relevant in cases where (4.16) is violated.

\subsubsection{Special bounds for (4.11)}

Below we shall look for stepsize-coefficients for boundedness using the representation of (4.11) in the form (1.2) with the matrices $S, T$ specified in Section 4.3.1.

For the matrix $T$ we have (2.1) (for all $\gamma>0$ ). To prove this, we consider the alternative ordering

$$
y_{2 i-1}=v_{k-1+i}, \quad y_{2 i}=u_{k-1+i} \quad(1 \leq i \leq N)
$$

which yields a representation of type (4.14) with strictly lower triangular matrices, say, $\underline{\mathrm{A}}, \underline{\mathrm{B}}$. The corresponding matrix $\underline{\mathrm{T}}=(I-\underline{\mathrm{A}})^{-1} \underline{\mathrm{B}}$ is also strictly lower triangular. With our original ordering, viz. (4.12), we thus have a matrix $T=V \underline{\mathrm{T}} V^{-1}$, where $V$ is a permutation matrix, and therefore (2.1) holds. To derive boundedness results it will be convenient to use the original ordering (4.12).

Substituting in (2.2) the expressions for $S$ and $T$, we arrive at

$$
R=K J, \quad P=\gamma K B, \quad K=(I-A+\gamma B)^{-1} .
$$

Because $P=V \underline{\mathrm{P}} V^{-1}$, with $\underline{\mathrm{P}}=\gamma \underline{\mathrm{T}}(I+\gamma \underline{\mathrm{T}})^{-1}$ and $\operatorname{spr}(\underline{\mathrm{P}})=0$, we have also $\operatorname{spr}(P)=0$.

Let $\check{K}_{0}=\left(I-\check{A}_{0}+\gamma \check{B}_{0}\right)^{-1}, \check{A}_{0}=A_{0}-\gamma b_{0} \hat{A}_{0}, \check{B}_{0}=B_{0}-\gamma b_{0} \hat{B}_{0}$. It can be seen that

$$
K=\left(\begin{array}{cc}
I-A_{0}+\gamma B_{0} & \gamma b_{0} I \\
-\hat{A}_{0}+\gamma \hat{B}_{0} & I
\end{array}\right)^{-1}=\left(\begin{array}{cc}
\check{K}_{0} & -\gamma b_{0} \check{K}_{0} \\
\left(\hat{A}_{0}-\gamma \hat{B}_{0}\right) \check{K}_{0} & \left(I-A_{0}+\gamma B_{0}\right) \check{K}_{0}
\end{array}\right)
$$

This gives

$$
R=\left(\begin{array}{cc}
\check{K}_{0} J_{0} & -\gamma b_{0} \check{K}_{0} J_{0} \\
\left(\hat{A}_{0}-\gamma \hat{B}_{0}\right) \check{K}_{0} J_{0} & \left(I-A_{0}+\gamma B_{0}\right) \check{K}_{0} J_{0}
\end{array}\right)
$$

Using the fact that lower triangular Toeplitz matrices commute, it is found that

$$
P=\gamma\left(\begin{array}{cc}
\left(B_{0}-\gamma b_{0} \hat{B}_{0}\right) \check{K}_{0} & b_{0} \check{K}_{0} \\
\left(\left(I-A_{0}\right) \hat{B}_{0}+\hat{A}_{0} B_{0}\right) \check{K}_{0} & b_{0}\left(\hat{A}_{0}-\gamma \hat{B}_{0}\right) \check{K}_{0}
\end{array}\right)
$$


We have

$$
S=\left(\begin{array}{cc}
\left(I-A_{0}\right)^{-1} J_{0} & O \\
\hat{A}_{0}\left(I-A_{0}\right)^{-1} J_{0} & J_{0}
\end{array}\right)
$$

By considering the upper-right blocks of $R, P, S$ and $P S,|P| S$ it can be seen that none of conditions (3.4)-(3.7) is fulfilled (for any $\gamma>0$ and all $N \geq 1$ ). Hence, Theorem 3.5 cannot be applied here directly so as to arrive at (4.3) with positive $\gamma$. However, we shall see below that a positive stepsize-coefficient for boundedness can be found by modifying the matrix $S$ and applying Theorem 3.9.

Let

$$
\tilde{x}_{i}=x_{i}-\gamma b_{0} x_{i+k}, \quad \tilde{x}_{i+k}=x_{i+k} \quad \text { for } i=1, \ldots, k .
$$

Then $x=V \tilde{x}$ with $V=\left(\begin{array}{cc}I & \gamma b_{0} I \\ O & I\end{array}\right)$. Below we shall deal with process (4.14) written in the equivalent form

$$
y=\tilde{\boldsymbol{S}} \tilde{x}+\Delta t \boldsymbol{T} \boldsymbol{F}(y),
$$

where $\tilde{S}=\left(\tilde{s}_{i j}\right)=(I-A)^{-1} J V=S V$. Defining $\tilde{R}=(I+\gamma T)^{-1} \tilde{S}$ (cf. (2.2)) we get in view of (4.19)

$$
\tilde{R}=K J V=\left(\begin{array}{cc}
\check{K}_{0} J_{0} & O \\
\left(\hat{A}_{0}-\gamma \hat{B}_{0}\right) \check{K}_{0} J_{0} & J_{0}
\end{array}\right)
$$

We now have $\tilde{R} \geq 0$ (for all $N \geq 1$ ) whenever

$$
P \geq 0 \quad(\text { for all } N \geq 1) \text {. }
$$

This leads directly to the following result.

Lemma 4.5. Consider $N$ consecutive steps of process (4.11) written in the form (4.23). Assume (3.14) and let F satisfy (4.1). Assume $\gamma>0$, (4.25) and $0<\Delta t \leq \gamma \cdot \tau_{0}$. Then the output vectors $y_{i}$ defined by (4.12) satisfy

$$
\left\|y_{i}\right\| \leq \tilde{\mu}_{i} \cdot \max _{1 \leq j \leq l}\left\|\tilde{x}_{j}\right\| \quad(1 \leq i \leq 2 N)
$$

with $\tilde{\mu}_{i}=\sum_{j}\left|\tilde{s}_{i j}\right|$.

Proof. To prove this lemma, we apply part (I) of Theorem 3.9 with $S$ replaced by $\tilde{S}$.

Consider $\tilde{\mu}=\max _{i} \tilde{\mu}_{i}=\|\tilde{S}\|_{\infty}$. Using (4.15) and (4.24), there follows after a little calculation that

$$
\tilde{S}=\left(\begin{array}{cc}
I & \gamma b_{0} I \\
\hat{A}_{0} & I-\check{A}_{0}
\end{array}\right)\left(\begin{array}{cc}
S_{0} & 0 \\
0 & S_{0}
\end{array}\right)
$$

with $S_{0}=\left(I-A_{0}\right)^{-1} J_{0}$. We find that $\tilde{\mu} \leq\left\|\left(I-A_{0}\right)^{-1} J_{0}\right\|_{\infty} \cdot \max \left\{1+\gamma b_{0}, 1+\sum_{j=1}^{k}\left(\left|\hat{a}_{j}\right|+\right.\right.$ $\left.\left.\left|\check{a}_{j}\right|\right)\right\}$. Due to the assumption of zero-stability we have $\sup _{N>1}\left\|S_{0}\right\|_{\infty}<\infty$, so that $\tilde{\mu}$ can be bounded, uniformly with respect to $N$.

Consider $\gamma>0$ such that (4.25) holds and let $0<\Delta t \leq \gamma \cdot \tau_{0}$. Then from Lemma 4.5 and (4.13), (4.22), it follows that

$$
\left\|u_{n}\right\| \leq \tilde{\mu} \cdot \max \left\{\sum_{j=1}^{k}\left(\left|\check{a}_{j}-\gamma \check{b}_{j}\right|+\left|\gamma \check{b}_{j}\right|\right), \sum_{j=1}^{k}\left(\left|\hat{a}_{j}-\gamma \hat{b}_{j}\right|+\left|\gamma \hat{b}_{j}\right|\right)\right\} \cdot \max _{0 \leq j \leq k-1}\left\|u_{j}\right\|
$$

for $k \leq n \leq k-1+N$, whenever $u_{n}$ is generated from $u_{0}, \ldots, u_{k-1} \in \mathbb{V}$ by applying (4.11) under assumption (4.1), where $\|\cdot\|$ is a seminorm on the vector space $\mathbb{V}$. Thus we arrive at the following theorem.

Theorem 4.6. Assume $\gamma>0$ is such that (4.25) holds. Then $\gamma$ is a stepsize-coefficient for boundedness of (4.11) (in the sense of Section 4.1). 


\subsubsection{Results for third order explicit two-step methods of the form (4.11)}

In this section we study method (4.11) with $k=2, u_{n} \approx u(n \Delta t), v_{n} \approx u((n-\kappa) \Delta t)$. Requiring order $p=3$ leaves 3 free parameters $a_{1}, \hat{a}_{1}, \kappa$ and the remaining coefficients can be computed by the formulas: $a_{2}=1-a_{1}, b_{0}=\left(4+a_{1}\right) /(6(1-\kappa)(2-\kappa)), b_{1}=(8-12 \kappa-$ $\left.(4-3 \kappa) a_{1}\right) /(6(1-\kappa)), b_{2}=\left(4-(5-3 \kappa) a_{1}\right) /(6(2-\kappa)), \hat{a}_{2}=1-\hat{a}_{1}, \hat{b}_{1}=2-\frac{\hat{a}_{1}}{2}-2 \kappa+\frac{\kappa^{2}}{2}$, $\hat{b}_{2}=-\frac{\hat{a}_{1}}{2}+\kappa-\frac{\kappa^{2}}{2}$. The method is zero-stable if and only if $a_{1} \in[0,2)$.

For these methods we will compute the maximal values of $\gamma$ such that $P \geq 0$ for all $N=1, \ldots, 1000$; it was verified that with larger $N$ the results did not differ anymore noticeably.

First we study the methods with $\kappa=0$, corresponding to the classical two-step predictorcorrector methods. The result is shown in the left panel of Figure 1. We note that there are no methods in this class for which the monotonicity condition (4.16) holds with $\gamma>0$. The displayed values of $\gamma$ for boundedness with these predictor-corrector methods are rather low; the maximal value is approximately 0.36 , corresponding to $a_{1} \approx 0.765, \hat{a}_{1} \approx 1.673$.
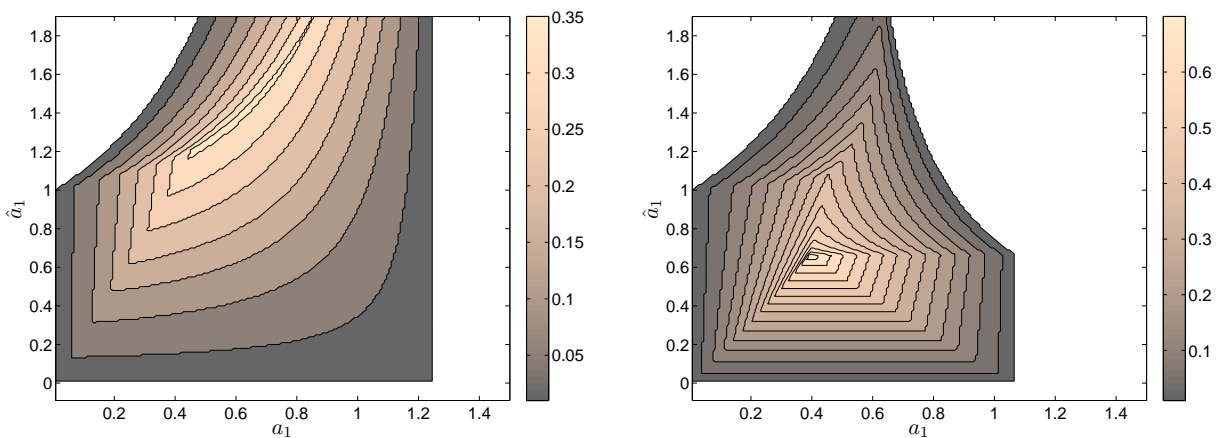

Figure 1: Maximal values $\gamma>0$ such that $P \geq 0$ for the methods (4.11) with $k=2$ of order $p=3$, with parameters $a_{1} \in[0,1.5]$ horizontally and $\hat{a}_{1} \in[-0.1,1.95]$ vertically. Left panel: standard predictor-corrector methods, $\kappa=0$. Right panel: hybrid methods with $\kappa=1-\frac{1}{3} \sqrt{3}$. Contour levels at $j / 20, j=0,1, \ldots$; for the 'white' areas, there is no positive $\gamma$.

A numerical search revealed that larger values of $\gamma$ can be found by allowing $\kappa \neq 0$. The right panel of Figure 1 shows the values of $\gamma$ with $\kappa=1-\frac{1}{3} \sqrt{3}$. The largest $\gamma \approx 0.73$ is found with $a_{1} \approx 0.392, \hat{a}_{1} \approx 0.667$ and this $\gamma$ is optimal within the whole class (4.11) with $k=2, p=3$.

Rather surprisingly, this method coincides with the method found in [22, Section 3.2.3] which is optimal with respect to the monotonicity condition (4.16). The latter method corresponds to $a_{1}=6 \sqrt{3}-10, \hat{a}_{1}=\frac{2}{3}$. These parameters coincide (up to four decimal digits) with the values for $a_{1}, \hat{a}_{1}$ obtained numerically by our search using condition (4.25), corresponding to the right panel in Figure 1. In fact, if $\hat{a}_{1} \leq \frac{2}{3}$ the monotonicity condition (4.16) seems to give the same $\gamma$ as the boundedness condition (4.25). If $\hat{a}_{1}>\frac{2}{3}$ then the method has some negative coefficient, so then there is no positive $\gamma$ for monotonicity with arbitrary starting values. But, as shown by Figure 1, for such $\hat{a}_{1}$ we can still have positive stepsize-coefficients $\gamma$ for boundedness.

\section{References}

[1] Butcher J.C. (1966): On the convergence of numerical solutions to ordinary differential equations, Math. Comp. 20, 1-10.

[2] Butcher J.C. (1987): The numerical analysis of ordinary differential equations, John Wiley, Chichester, UK. 
[3] Butcher J.C. (2003): Numerical methods for ordinary differential equations, John Wiley, Chichester, UK.

[4] Ferracina L., Spijker M.N. (2004): Stepsize restrictions for the total-variationdiminishing property in general Runge-Kutta methods, SIAM J. Numer. Anal. 42, 1073-1093.

[5] Gottlieb S, Ketcheson D.I, Shu C.-W. (2009): High order strong stability preserving time discretizations, J. Scientif. Computing 38, 251-289.

[6] Gottlieb S., Shu C.-W., Tadmor E. (2001): Strong stability-preserving high-order time discretization methods, SIAM Review 43, 89-112.

[7] Hairer E., Wanner G. (1996): Solving ordinary differential equations. II. Stiff and differential-algebraic problems, Springer-Verlag, Berlin.

[8] Hairer E., Nørsett S.P., Wanner G. (1987): Solving ordinary differential equations. I. nonstiff problems, Springer-Verlag, Berlin.

[9] Higueras I. (2004): On strong stability preserving time discretization methods, Journ. Scientif. Computing 21, 193-223.

[10] Higueras I. (2005): Representations of Runge-Kutta methods and strong stability preserving methods, SIAM J. Numer. Anal. 43, 924-948.

[11] Horn R.A., Johnson C.R. (1988): Matrix analysis, Cambridge University Press, Cambridge.

[12] Huang, C. (2009): Strong stability preserving hybrid methods. Appl. Num. Meth. 59, 891-904.

[13] Hundsdorfer W., Mozartova A.S., Spijker M.N. (2009): Stepsize conditions for boundedness in numerical initial value problems, SIAM J. Numer. Anal. 47, 3797-3819.

[14] Hundsdorfer W., Ruuth S.J. (2003): Monotonicity for time discretizations, Procs. Dundee Conference 2003, pp. 85-94. Eds. D.F. Griffiths, G.A. Watson, Report NA/217, Univ. Dundee.

[15] Hundsdorfer W., Ruuth S.J. (2006): On monotonicity and boundedness properties of linear multistep methods, Math. Comp. 75, 655-672.

[16] Hundsdorfer W., Ruuth S.J., Spiteri R.J. (2003): Monotonicity-preserving linear multistep methods, SIAM J. Numer. Anal. 41, 605-623.

[17] Hundsdorfer W., Verwer J.G. (2003): Numerical solution of time-dependent advectiondiffusion-reaction equations, Springer Ser. Comp. Math., Vol 33, Springer (Berlin)

[18] LeVeque R. J. (2002): Finite volume methods for hyperbolic problems, Cambridge University Press.

[19] Ruuth S.J., Hundsdorfer W. (2005): High-order linear multistep methods with general monotonicity and boundedness properties, J. Comput. Phys. 209, 226-248.

[20] Shu C.-W., Osher S. (1988): Efficient implementation of essentially nonoscillatory shock-capturing schemes, J. Comput. Phys. 77, 439-471.

[21] Spijker M.N (1983): Contractivity in the numerical solution of initial value problems, Numer. Math. 42, 271-290.

[22] Spijker M.N. (2007): Stepsize conditions for general monotonicity in numerical initial value problems, SIAM J. Numer. Anal. 45, 1226-1245. 